\title{
Modeling Influence In Group Decision Making
}

\author{
Luis G. Pérez • Francisco Mata - Francisco Chiclana - Gang Kou • \\ Enrique Herrera-Viedma
}

Received: date / Accepted: date

\begin{abstract}
Group decision making has been widely studied since group decision making processes are very common in many fields. Formal representation of the experts' opinions, aggregation of assessments or selection of the best alternatives have been some of main areas addressed by scientists and researchers. In this paper, we focus on another promising area, the study of group decision making processes from the concept of influence and social networks. In order to do so, we present a novel model that gathers the experts' initial opinions and provides a framework to represent the influence of a given expert over the other(s). With this proposal it is feasible to estimate both the evolution of the group decision making process and the final solution before carrying out the group discussion process and consequently foreseeing possible actions.
\end{abstract}

Keywords Group decision making · Aggregation operators · Social network · Influence.

Luis G. Pérez and Francisco Mata

Department of Computer Science

University of Jaén, 23071 Jaén, Spain

E-mail: lgonzaga@ujaen.es, fmata@ujaen.es

Francisco Chiclana

Centre for Computational Intelligence (CCI)

Faculty of Technology, De Montfort University, LE1 9BH, UK.

E-mail: chiclana@dmu.ac.uk

Gang Kou

School of Business Administration

Southwestern University of Finance and Economics, Chengdu, 611130 China

E-mail: kougang@swufe.edu.cn

Enrique Herrera-Viedma

Department of Computer Science

University of Granada, 18071 Granada, Spain.

E-mail: viedma@decsai.ugr.es

\section{Introduction}

Decision making can be defined as a set of activities whose aim is to find satisfactory solutions for a given problem. This problem usually involves the analysis of a finite set of alternatives. From this analysis, decision makers, judges or expert(s) should decide which of the alternatives is the best one to solve an issue. Decision making can be approached from two points of view:

- Individual decision making: in which an expert must provide the best alternative from a set of possible solution, either evaluating the utility of each alternative or assessing a set of criteria that described them.

- Group decision making: in which a group of experts must reach a solution to a given problem.

Although decision making has been widely studied, only a few areas have drawn the attention of researchers. For instance, the formal representation of the experts' opinions $[20,21,23,34,39,41,42]$, the aggregation of the information $[33,38]$ and, the selection of the best alternative(s) $[4,28]$ have been developed in depth by many authors.

Another promising field is the consensus in decision making problems $[2,5,6,11,16,19,25,26]$. In this kind of problems, the aim is to reach a given course of action that the majority of experts approve and that the minority agree to consent. In such a way, we avoid that the minority feel that their opinions are not taken into account.

In this paper, we focus on another interesting area related to group decision making. We present a model based on 'influence' concept that let us estimate both the evolution of the group decision making process and the final solution from the experts' initial opinions. 
The utility of this field is propitious since on the one hand, we can predict beforehand the final decision of a given problem and, on the other hand, this model will let us know how the opinion of one expert affects their partners' opinions throughout the process.

Few papers have dealt with the modeling of influences between people through discussion and exchanges of opinions $[14,15]$ and our aim is to apply these studies to group decision making processes.

A Group Decision Making (GDM) process usually entails an activity in which experts interact with each other in order to reach a final solution. These interactions consist of an exchange of opinions and information. Because of these interactions, experts with a wider background, experience and knowledge will be recognized and empowered with a higher status. It is very interesting to notice that this status reflects the influence of a given expert over the other(s), and therefore, if we know the experts' initial opinions, if we find a suitable representation for the influence among experts and if we gather this information, we may be able to predict both the evolution of the experts' opinions and their final decisions. In order to represent this influence network we propose the use of so-called social network analysis.

Social network analysis $[37,43]$ studies relationships among social entities or actors as well as patterns and implications of these relationships. These relationships may be of many sorts: economic, political, interactional, affective, etc. Social network analysis is based on the idea that actors and their actions are interdependent and the network structural environment provides opportunities for or constraints on individual actions.

Using social network analysis in GDM problems can produce important benefits since it allows us to model and study the relationship between experts, and therefore, infer or estimate the influence among themselves. The aim of this paper is to model this situation.

The structure of this paper is the following: First of all, in section 2 we review some preliminaries, then the model is presented in section 3. Finally, section 4 shows an example and the conclusions are pointed out in section 5 .

\section{Preliminaries}

In this section, we sum up some aspects needed to understand our proposal such as group decision making, consistency and social influence networks theory.

\subsection{Group Decision Making}

In Group Decision Making problems (GDM), a group of experts must decide which alternative(s) is the best one to deal with an issue. For this reason, each one of them provides an assessment of every possible alternative. To represent these judgements, some structures are proposed. The most common ones are:

- A preference ordering of alternatives [38]: in this case, experts provide their preferences on a set of alternatives as an ordered vector of alternatives, from the best one to the worst one.

- Utility vectors [23,41]: with this representation experts provide their preferences as a set of utility values for each alternative. The greater the value is, the more preferred the alternative is.

- Preference relations [18, 20, 21, 39]: based on the idea of pairwise comparisons, experts' preferences are described by means of preference relations in which each value represents the preference of one alternative over the other.

Once experts have provided their opinion, we must accomplish the selection of the best alternatives. This process usually involves two phases $[1,32,35,44]$ :

- An aggregation phase: in this step, experts' opinions are aggregated to obtain a global opinion.

- A selection phase: in this phase the best alternative(s) is chosen and presented as the decision making solution.

One of the most used preference structure in GDM is the preference relation. In preference relations, we find two kind of interpretation regarding preference assessments, it can be additive reciprocal and multiplicative reciprocal. In this contribution, we have applied the additive preference relation and its definition is the following one:

Definition 1 [3, 9,30]: An additive reciprocal fuzzy preference relation $R$ on a finite set of alternatives $X$ is a fuzzy relation in $X \times X$ with membership function $\mu_{R}: X \times X \rightarrow[0,1], \mu_{R}\left(x_{i}, x_{j}\right)=r_{i j}$, verifying

$$
r_{i j}+r_{j i}=1 \forall i, j \in\{1, \ldots, n\} .
$$

When cardinality of $X$ is small, the reciprocal fuzzy preference relation may be conveniently denoted by the matrix $R=\left(r_{i j}\right)$ and the following interpretation is also usually assumed:

$-r_{i j}=1$ indicates the maximum degree of preference for $x_{i}$ over $x_{j}$.

- $\left.r_{i j} \in\right] 0.5,1\left[\right.$ indicates a definite preference for $x_{i}$ over $x_{j}$. 
- $r_{i j}=0.5$ indicates indifference between $x_{i}$ and $x_{j}$.

$\left.-r_{i j} \in\right] 0,0.5\left[\right.$ indicates a definite preference for $x_{j}$ over $x_{i}$.

Another important aspect related to GDM is opinion aggregation in order to compute a global opinion over a set of alternatives. Many operators have been proposed to carry out this computation highlighting the family of OWA operators.

In this section, the OWA[46], IOWA[49] and I-IOWA operators are introduced [10].

Definition 2 An OWA operator of dimension $n$ is a mapping $\phi: \mathbb{R}^{n} \rightarrow \mathbb{R}$, which has an associated set of weights $W=\left(w_{1}, \cdots, w_{n}\right)^{T}$ to it, such that $w_{i} \in[0,1]$, $\sum_{i=1}^{n} w_{i}=1$

$\phi(a)=\phi\left(p_{1}, \cdots, p_{n}\right)=\sum_{i=1}^{n} w_{i} p_{\sigma(i)}$

and $\sigma:\{1, \cdots, n\} \longrightarrow\{1, \cdots, n\}$ is a permutation function such that $p_{\sigma(i)} \geq p_{\sigma(i+1)}, \forall i=1, \cdots, n-1$.

In order to obtain the associated weighting vector, we use the method proposed by Yager $[45,46]$ to give semantic or meaning to weights by means of quantifier guided aggregations. To implement the concept of fuzzy majority in the aggregation phase, we use a fuzzy linguistic quantifier [51], which indicates the proportion of satisfied criteria "necessary for good solution" [47] (see the appendix A for further details). In case of a regular increasing monotone (RIM) quantifier Q, the weights are computed using the expression [46]:

$$
w_{i}=Q\left(\frac{i}{n}\right)-Q\left(\frac{i-1}{n}\right), i=1, \ldots, n .
$$

Mitchell and Estrakh in [27] described a modified OWA operator in which the input arguments are not re-arranged according to their values but rather using a function of the arguments. Inspired by this work, Yager and Filev introduced in [49] a more general type of OWA operator, which they named the Induced OWA (IOWA) operator:

Definition 3 : An IOWA operator of dimension $n$ is a mapping $\Phi_{W}:(\mathbb{R} \times \mathbb{R})^{n} \longrightarrow \mathbb{R}$, which has an associated set of weights $W=\left(w_{1}, \cdots, w_{n}\right)$ to it, so that $w_{i} \in$ $[0,1], \sum_{i=1}^{n} w_{i}=1$,

$$
\Phi_{W}\left(\left\langle u_{1}, p_{1}\right\rangle, \ldots,\left\langle u_{n}, p_{n}\right\rangle\right)=\sum_{i=1}^{n} w_{i} p_{\sigma(i)},
$$

and $\sigma:\{1, \ldots, n\} \longrightarrow\{1, \ldots, n\}$ is a permutation function such that $u_{\sigma(i)} \geq u_{\sigma(i+1)}, \forall i=1, \ldots, n-1$.
In the above definition the reordering of the set of values to aggregate, $\left\{p_{1}, \ldots, p_{n}\right\}$, is induced by the reordering of the set of values $\left\{u_{1}, \ldots, u_{n}\right\}$ associated to them, which is based upon their magnitude. Yager and Filev called the vector of values $\left(u_{1}, \ldots, u_{n}\right)$, the order inducing vector and $\left\{p_{1}, \ldots, p_{n}\right\}$, the values of the argument variable $[49,50,48]$. Thus, the main difference between the OWA operator and the IOWA operator is the reordering step of the argument variable. In the case of OWA operator this reordering is based upon the magnitude of the values to be aggregated, while in the case of IOWA operator an order inducing vector is used as the criterion to induce that reordering.

In many cases, each expert $e_{k} \in E$ is assigned an importance degree $u_{k}$ to him/her, which without loss of generality can be assumed to belong to the unit interval, i.e. $u_{i} \in[0,1] \forall i$, and that there is some $i$ such that $u_{i}=1$. This can always be assured by taking the normalised importance degrees that result when dividing them by the maximum importance degree. Thus, importance degree can be interpreted as a fuzzy set membership function, $\mu_{I}: E \rightarrow[0,1]$, in such a way that $\mu_{I}\left(e_{k}\right)=u_{k} \in[0,1]$ denotes the importance degree of the opinion provided by the expert $e_{k}$. In the area of quantifier guided aggregations, Yager in [47] presents a procedure to evaluate the overall satisfaction of $Q$ important criteria (experts) by an alternative $x$. In this procedure, once the satisfaction values to be aggregated have been ordered, the weighting vector associated to an OWA operator using a linguistic quantifier $Q$ is calculated following the expression

$w_{k}=Q\left(\frac{S(k)}{S(n)}\right)-Q\left(\frac{S(k-1)}{S(n)}\right)$

being $S(k)=\sum_{l=1}^{k} u_{\sigma(l)}$, and $\sigma$ the permutation used to produce the ordering of the values to be aggregated. This approach for the inclusion of importance degrees associates a zero weight to those experts with zero importance degree. This procedure was extended by Yager to the case of induced aggregation [48] and later by Chiclana et al. [10] to introduce the Importance IOWA (I-IOWA) operator. In this case, each component in the aggregation consist of a tuple $\left(u_{k}, p_{k}\right)$ where $p_{k}$ is the argument value to aggregate and $u_{k}$ is the importance weight value associated to $p_{k}$ and also the order inducing value. This is summarised in the following definition:

Definition 4 Let $E=\left\{e_{1}, \ldots, e_{m}\right\}$ be a set of experts and $U=\left(u_{1}, \ldots, u_{m}\right) \in[0,1]^{n}$ the vector of their associated importance degrees. An I-IOWA operator of dimension $n, \Phi_{W}^{I}$, is an IOWA operator whose order inducing vector is the vector of importance degrees and 
following associated set of weights $W=\left(w_{1}, \cdots, w_{n}\right)$ :

$$
\Phi_{W}^{I}\left(\left\langle u_{1}, p_{1}\right\rangle, \ldots,\left\langle u_{n}, p_{n}\right\rangle\right)=\sum_{i=1}^{n} w_{i} p_{\sigma(i)},
$$

with

$w_{k}=Q\left(\frac{S(k)}{S(n)}\right)-Q\left(\frac{S(k-1)}{S(n)}\right)$,

where $S(k)=\sum_{l=1}^{k} u_{\sigma(l)}$, and $\sigma$ is the permutation such that $u_{\sigma(i)} \geq u_{\sigma(i+1)}, \forall i=1, \ldots, n-1$.

With the I-IOWA operator, we accomplish the selection phase. To do so, some authors [10] have proposed the use of a quantifier-guided choice degree of alternatives, a dominance degree. With this degree it is computed the best acceptable alternative for the majority (Q).

Definition 5 : The quantifier-guided dominance degree, $Q G D D_{i}$, quantifies the dominance that one alternative has over all the others and is defined as follows:

$$
Q G D D_{i}=\phi_{Q}\left(p_{i 1}^{c}, p_{i 2}^{c}, \ldots, p_{i n}^{c}\right) .
$$

So, for each alternative, $x_{i}$, we obtain a value that quantify the dominance that this alternative has over all the others in a fuzzy majority sense.

With this degree we can sort out the alternatives and choose the best one(s),

$$
X^{\text {sol }}=\left\{x_{i} \mid x_{i} \in X, Q G D D_{i}=\sup _{j} Q G D D_{j}\right\} .
$$

An example of how to use these operators in GDM problems is shown in appendix B.

\subsection{Consistency on Preference Relations}

Although preference relations are one of the most used preference structures, these also have some disadvantages. First of all, the way of providing preferences limits experts in their global perception of the alternatives and as a consequence, the provided preferences could be inconsistent.

In [8] the U-Consistency Method, useful to tackle the problem of incomplete information in reciprocal fuzzy preference relations, is presented. This method is based on the modeling of consistency of preferences via a self-dual almost continuous uninorm operator[7] and computes the missing pairwise preference values from the known ones with the aim of maintaining or maximising the expert's global consistency. This process is applied as well to complete preference relations as it is our case. With this process, we can assure that the values provided by the expert are compatible with the rest of information and, moreover, we can replace those ones that are not compatible with the estimated values.

In order to deal with the inconsistency, we must understand that this representation assumes three fundamental and hierarchical levels of rationality:

- The first level of rationality requires indifference between any alternative $x_{i}$ and itself.

- The second one requires that if an expert prefers $x_{i}$ to $x_{j}$, that expert should not simultaneously prefer $x_{j}$ to $x_{i}$. This asymmetry condition is viewed as an 'obvious' condition/criterion of consistency for preferences. This rationality condition is modeled by the property of reciprocity in the pairwise comparison between any two alternatives, which is seen by Saaty [36] as basic in making paired comparisons.

- Finally, the third one is associated with the transitivity in the pairwise comparison among any three alternatives.

A preference relation verifying the third level of rationality is usually called a consistency preference relation and any property that guarantees the transitivity of the preferences is called a consistency property. However, many properties or conditions have been suggested in the literature of fuzzy preference to model the third level of rationality. Among these properties we can cite: (restricted) max-min transitivity, restricted max-max transitivity, additive transitivity, multiplicative transitivity $[7,12,40]$, and we have used Tanino's multiplicative transitivity [40]

Next, we review some definitions to understand how to improve the consistency of a relationship.

Definition 6 [7]: Let $U$ be a representable uninorm operator with strong negation $N(x)=1-x$ and let $R$ be an additive reciprocal fuzzy preference relation on a finite set of alternatives $X, \mu_{R}: X \times X \rightarrow[0,1]$, where $\mu_{R}\left(x_{i}, x_{j}\right)=r_{i j}$ represents the preference degree of the alternative $x_{i}$ over $x_{j}$, then the fuzzy preference relation $R$ on a finite set of alternatives is consistent with respect to $U(U-$ Consistent $)$ if

$\forall i, j, k:\left(r_{i j}, r_{k j}\right) \notin\{(0,1),(1,0)\} \Rightarrow r_{i j}=U\left(r_{i k}, r_{k j}\right)$.

Tanino's multiplicative transitivity property [40] under reciprocity is the restriction to the region $[0,1] \times$ $[0,1] \backslash\{(0,1),(1,0)\}$ of the following well known andlike representable uninorm [22]

$$
U(x, y)= \begin{cases}0, & (x, y) \in\{(0,1),(1,0)\} \\ \frac{x y}{x y+(1-x)(1-y)} & \text { otherwise }\end{cases}
$$

In order to improve the quality of the information provided by the experts we use this transitivity. Given 
a fuzzy preference relation $R$ and $U$ a representable uninorm (with $N(X)=1-x$ ), the preference value $r_{i k}$ $(i \neq k)$ is partially U-estimated using an intermediate alternative $x_{j}$ such that $\left(r_{i j}, r_{j k}\right) \notin\{(0,1),(1,0)\}$ as follows:

$$
u r_{i k}^{j}=U\left(r_{i j}, r_{j k}\right) \text {. }
$$

The average of the partially $U$-estimated values obtained using all possible intermediate alternatives can be seen as the global consistency based estimated value

$$
u r_{i k}=\frac{\sum_{j \in R_{i k}^{01}} u r_{i k}^{j}}{\# R_{i j}^{01}},
$$

where $R_{i k}^{01}=\left\{j \neq i, j \mid\left(r_{i j}, r_{j k}\right) \notin\{(0,1),(1,0)\}\right\}$.

With this definition, we estimate the missing values of a relationship or as in our case to improve the consistency of given relationship.

In our model, it is also very important to measure the U-Consistency of a preference relation. A preference relation is completely U-Consistency if $u r_{i k}$ agrees with $r_{i k}\left(u r_{i k}=r_{i k}\right)$. However, experts are not always fully consistent and because of this, it is possible to calculate the difference between the actual value $r_{i k}$ and the the estimated one $u r_{i k}$ :

$$
\varepsilon u r_{i k}=\left|u r_{i k}-r_{i k}\right| \text {. }
$$

From this formula, it is defined the U-Consistency level associated with a preference value $r_{i k}$, and then, the $U$-consistency level of the whole reciprocal fuzzy preference relation $R$.

Definition 7 [8]: Given $U$ a representable uninorm with $N(x)=1-x$, the $U$-consistency level associated with a preference value $r_{i k}$ is defined as:

$$
U C L_{i k}=1-\varepsilon u r_{i k},
$$

Definition 8 [8]: Given $U$ a representable uninorm with $N(x)=1-x$, the $U$-consistency level of a fuzzy preference relation $R$ measured in [0,1] is given as follows:

$$
U C L_{R}=\frac{\sum_{i, j=1, i \neq k}^{n} U C L_{i k}}{n(n-1)} .
$$

\subsection{Social Influence Networks Theory}

Social Influence Network Theory began with French's formal theory of social power [13] and it has been developed by several authors $[14,15,17]$. The aim of this theory is to model the process of influence among actors belonging to the same social network. The following expression is a recursive definition of the influence process:

$$
y^{(t)}=A W y^{(t-1)}+(I-A) y^{(1)}
$$

for $t=2,3, \ldots$, where $y^{(1)}$ is an $N \times 1$ vector of actors' initial opinions on an issue, $y^{(t)}$ is an $N \times 1$ vector of actors' opinions a time $t, W=\left[w_{i j}\right]$ is an $N \times N$ matrix of interpersonal influences $\left(0 \leq w_{i j} \leq 1, \sum_{j}^{N} w_{i j}=1\right)$, and $A=\operatorname{diag}\left(a_{11}, a_{22}, \ldots, a_{N N}\right)$ is an $N \times N$ diagonal matrix of actors' susceptibilities to interpersonal influence on the issue $\left(0 \leq a_{i i} \leq 1\right)$.

This model is defined from the following assumptions [15]:

1. Cognitive Weighted Averaging: Actors are assumed to form their revised opinions through a weighted averaging of influences on them. This influence is not only exogenous because of the other actors' opinions, but also endogenous from their own initial opinion. The relative weight of the endogenous and exogenous influences for each actor is determined by $A=\left[a_{i i}\right]$, the coefficients of susceptibility to social influence.

2. Fixed Social Structure: The social structure of the group of actors, the network, is represented by the matrix $W$, is assumed to be fixed during the entire process of opinion formation.

3. Determinism: Given the direct influence matrix $W$ and the group members' initial opinion $y^{(1)}$, the subsequent opinion changes in the group are completely determined.

4. Continuance: The process of opinion formation in the group continues until all changes of opinions that may occur have played themselves out.

5. Decomposability: The opinion formation process can be divided into time periods, defined by the times $t=1,2,3, \ldots$, that may not be of the same length in real time.

6. Simultaneity: In each time period, simultaneous linear equations yield an accurate prediction of all the influence events that occur during that period.

If $W$ is known, then $A$ is computed as:

$$
a_{i i}=1-w_{i i}
$$

Assuming the process reaches an equilibrium, then $\lim _{t \rightarrow \infty} y^{(t)}=y^{(\infty)}=k<\infty$ and the original equation becomes:

$$
y^{(\infty)}=V y^{(1)}
$$

where $V$ describes the total interpersonal effects that transform initial opinions into final opinions. Then $V$ is calculated by using the following formula:

$$
V=(I-A W)^{-1}(I-A)
$$




\section{Modeling Influence in Group Decision Making}

In order to model an influence network in a GDM process, we propose to carry out the following phases:

1. Providing information: Experts' opinions are provided by means of reciprocal fuzzy preference relations. However, as we said beforehand, one of the disadvantages of this structure is that experts do not always provide consistent assessments. Thus, we need to improve the consistency of this information by means of a U-Consistency Based Method [8]. This method was originally presented to estimate missing pairwise preference values, however, this method is also used to improve the consistency of a given relationship. Finally, we transform the preference relation into utility vectors.

2. Modeling influence: In this phase we apply an influence model to estimate the evolution of the experts' opinions with regards to the other experts' opinions. Experts are supposed to discuss their opinion between them, depending on the influence between them, their opinions are modified. In order to model this, we use the model reviewed in section 2.3. In this model, the influence between experts is modeled by means of a social influence network. From this model, and with the experts' opinions, it is possible to infer the experts' final opinions.

3. Obtaining the solution: Once that final opinions have been calculated, the solution is computed. To do so, experts' utility vectors are aggregated by means of an I-IOWA operator. The I-IOWA is a weighted aggregation operator in which the information is aggregate based on the importance of the information sources. In our proposal, this importance is obtained by computing the consistency of the information that was initially provided by the experts. The more consistent it is, the more important the information is.

A representation of model's phases can be seen in Figure 1. In the following subsections we explain thoroughly these phases.

\subsection{Providing the information}

In this phase, a group of experts $E=\left\{e_{1}, \ldots, e_{m}\right\}$ provide their opinions about a set of alternatives $X=$ $\left\{x_{1}, \ldots, x_{n}\right\}$ by means of reciprocal preference relations. The use of these representation provides some advantages since they allow experts to focus exclusively on two alternatives at a time.

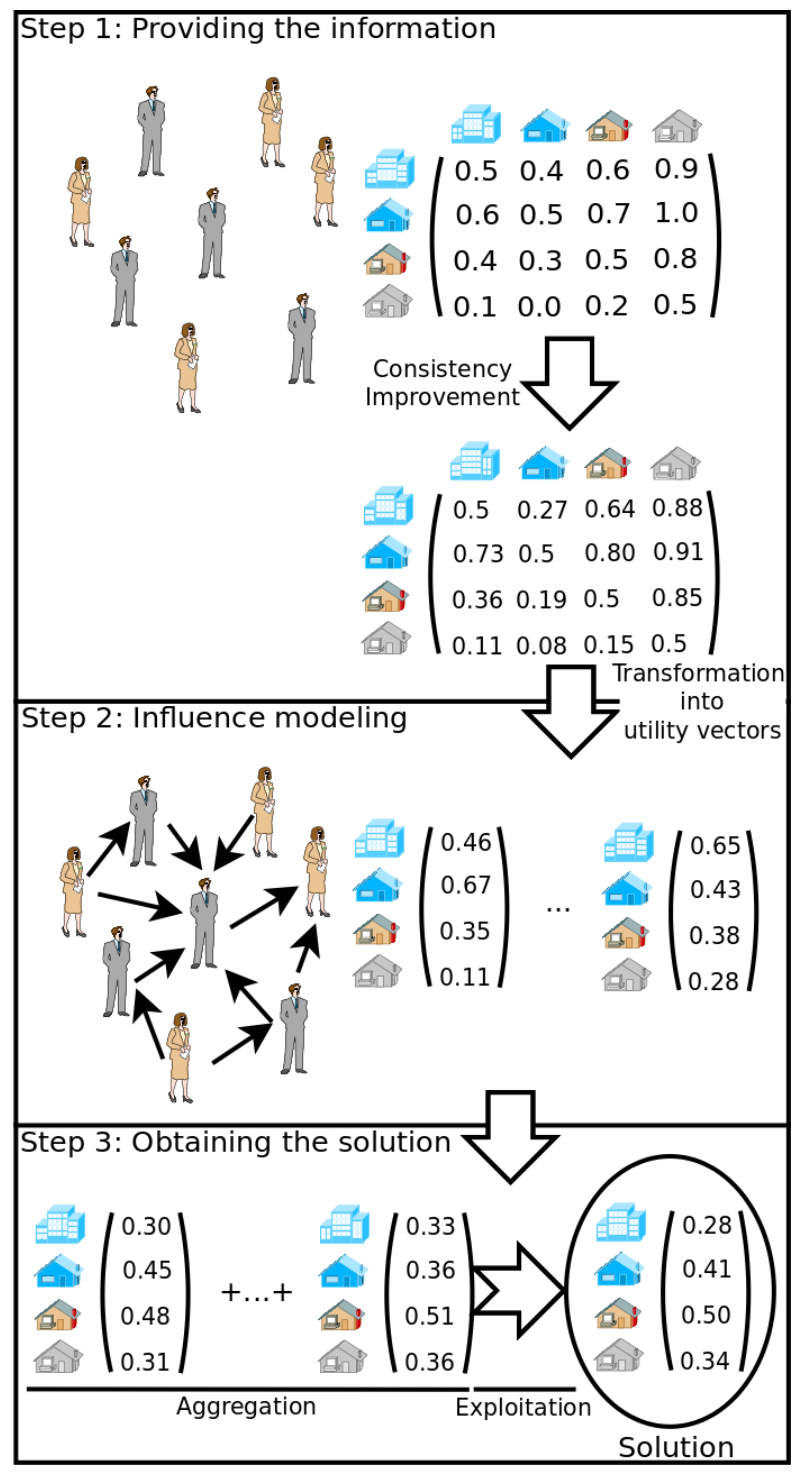

Fig. 1 Model's phases

Once we have the information, it is applied the consistency model reviewed in section 2.2 in order to improve the information provided by the experts.

\section{Example 1:}

Let $P_{1}$ be the opinion of the expert $e_{1}$ :

$$
P_{1}=\left(\begin{array}{ccccccc}
0.5 & 0.4 & 0.6 & 0.9 & 0.7 & 0.8 \\
0.6 & 0.5 & 0.7 & 1 & 0.8 & 0.9 \\
0.4 & 0.3 & 0.5 & 0.8 & 0.6 & 0.7 \\
0.1 & 0 & 0.2 & 0.5 & 0.3 & 0.4 \\
0.3 & 0.2 & 0.4 & 0.7 & 0.5 & 0.6 \\
0.2 & 0.1 & 0.3 & 0.6 & 0.4 & 0.5
\end{array}\right)
$$


then the preference relation obtained after applying the $U$-consistency method is:

$$
P_{1}^{\prime}=\left(\begin{array}{cccccc}
0.5 & 0.27 & 0.64 & 0.89 & 0.74 & 0.82 \\
0.73 & 0.5 & 0.8 & 0.92 & 0.85 & 0.89 \\
0.36 & 0.2 & 0.5 & 0.85 & 0.62 & 0.74 \\
0.11 & 0.083 & 0.15 & 0.5 & 0.2 & 0.27 \\
0.26 & 0.15 & 0.38 & 0.8 & 0.5 & 0.64 \\
0.18 & 0.11 & 0.26 & 0.73 & 0.36 & 0.5
\end{array}\right)
$$

Some of the obtained results are the following:

$$
\begin{gathered}
u r_{12}=\frac{u r_{12}^{3}+u r_{12}^{4}+u r_{12}^{5}+u r_{12}^{6}}{4}=\frac{1.08}{4}=0.27 . \\
u r_{13}=\frac{u r_{13}^{2}+u r_{13}^{4}+u r_{13}^{5}+u r_{13}^{6}}{4}=\frac{2.56}{4}=0.64 . \\
u r_{65}=\frac{u r_{65}^{1}+u r_{65}^{2}+u r_{65}^{3}+u r_{65}^{4}}{4}=\frac{1.44}{4}=0.36 .
\end{gathered}
$$

\subsection{Modeling influence}

Once experts' opinions have been transformed, we model the influence between experts. Experts are willing to discuss their decisions with each other. Because of these interactions, their opinions are influenced by other experts' opinions and they also influence the other ones. To apply the model summarized in section 2.3 we accomplish the following steps:

1. Transform the preference relationships into utility vectors.

2. Apply the influence model for each experts' opinion.

\subsubsection{Transform the preference relations into utility vectors}

In order to carry out the next step, information must be transformed into utility vectors firstly. To do so, we propose to use a quantifier-guided choice degree of alternatives, a dominance degree, to compute the best acceptable alternative for the majority $(Q)$. The quantifierguided dominance degree $Q G D D_{i}$ was reviewed in section 2.1 .

At the end of this step we have $m$ utility vectors, each one with the evaluation of the expert $i$ of the $n$ alternatives

$$
U_{i}=\left(\begin{array}{c}
u_{i 1} \\
\cdots \\
u_{i n}
\end{array}\right), i=1, \ldots, m .
$$

\subsubsection{Apply the influence model for each experts'} opinion

In this step our aim is to model how the experts' opinions evolve by the interaction of each other. Experts may differ in some of the opinions and it is expected that they talk each other in order to clarify, defend and modify their views.

As have been seen in section 2.3 we need to know the experts' initial opinions and the matrix $W$. The influence model is applied on one alternative, however in group decision making, experts provide their opinions about a set of alternatives $X=\left\{x_{1}, \ldots, x_{n}\right\}$. Therefore we have to decompose it in $n$ problems, each one for each alternative.

First of all, we need to compute the matrix $V$ as we explained in section 2.3:

$$
V=(I-A W)^{-1}(I-A)
$$

and then, we apply the influence model for each alternative:

$$
y_{x_{i}}^{(\infty)}=V y_{x_{i}}^{(1)}, i=1, \ldots, n
$$

We must realize the representation of the information used by the influence model is not the same as the representation used by the experts to provide their opinions. However, this information is easily transformed since $y_{x_{1}}^{(1)}$ is the initial opinion of all the experts regarding the alternative $x_{1}, y_{x_{2}}^{(1)}$ is the initial opinion of all the experts regarding the alternative $x_{2}$ and so on. Therefore, after transforming this information, we obtain:

$$
y_{x_{i}}^{(1)}=\left(\begin{array}{c}
u_{1 i} \\
\ldots \\
u_{m i}
\end{array}\right), i=1, \ldots, n .
$$

Once we have applied the influence model, we obtain the experts' final opinions:

$$
y_{x_{i}}^{(\infty)}=\left(\begin{array}{c}
y_{1 i}^{(\infty)} \\
\ldots \\
y_{m i}^{(\infty)}
\end{array}\right), i=1, \ldots, n
$$

and we easily use this information to obtain the final vectors $U_{1}^{(\infty)}, \cdots, U_{m}^{(\infty)}$ :

$$
U_{i}^{(\infty)}=\left(\begin{array}{c}
y_{i 1}^{(\infty)} \\
\cdots \\
y_{i n}^{(\infty)}
\end{array}\right), i=1, \ldots, m
$$

All these operations are described in depth in the following example. 
Example 2 : Let $E=\left\{e_{1}, e_{2}, e_{3}\right\}$ be the group of experts and let $X=\left\{x_{1}, x_{2}, x_{3}\right\}$ be the group of alternatives. Experts express their assessment by means of preference relations which have been transformed into utility vectors:

$$
U_{1}=\left(\begin{array}{c}
0.3 \\
0.4 \\
0.1
\end{array}\right), \cdots, U_{2}=\left(\begin{array}{l}
0.1 \\
0.2 \\
0.4
\end{array}\right), \cdots, U_{3}=\left(\begin{array}{l}
0.6 \\
0.2 \\
0.3
\end{array}\right)
$$

In order to apply the influence model, the model is applied to each alternative. In this example, we work with alternative $x_{1}$. First of all, it is obtained $y_{x_{1}}^{(1)}$ from the information provided by the experts:

$$
y_{x_{1}}^{(1)}=\left(\begin{array}{l}
0.3 \\
0.1 \\
0.6
\end{array}\right)
$$

where 1 is the initial period and $0.3,0.1$, and 0.6 are the $e_{1}$ 's initial assessment, $e_{2}$ 's initial assessment, and $e_{3}$ 's initial assessment about alternative $x_{1}$ respectively.

Next we define the matrix that represents the interpersonal influence of the group of experts (see Figure $2)$ :

$$
W=\left(\begin{array}{ccc}
0.2 & 0.2 & 0.6 \\
0.3 & 0.3 & 0.4 \\
0 & 0.2 & 0.8
\end{array}\right)
$$

The main diagonal of the matrix $W$, that is, $w_{i i}$, represents the experts' susceptibility to interpersonal influence. For instance, if an expert has $w_{i i}=1$ that means that this person is not subjected to any interpersonal influence. For example, $w_{12}=0.2$ indicates the direct relative influence of the expert 2 on expert 1 is 0.2 .

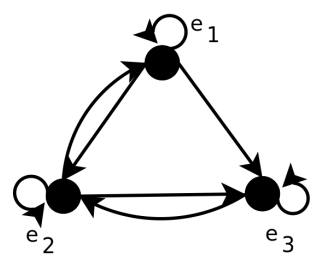

Fig. 2 Graph representation of matrix W

Now we apply the model. First of all, we calculate $V$ and we obtain:

$$
V=\left(\begin{array}{lll}
0.25 & 0.09 & 0.66 \\
0.07 & 0.41 & 0.52 \\
0.00 & 0.02 & 0.98
\end{array}\right)
$$

Matrix $V$ represents the network influence on every experts. It is very important to realize that $V$ and $W$ are two different matrices and represent different aspect of interpersonal influence among experts. $W$ represents the distribution of the direct relative interpersonal influence among experts. However, $V$, takes into account all the flows of interpersonal influence, both direct and indirect. For instance, $v_{12}=0.09$ and that indicates that the 9 percent of experts 1's final opinion is determined by experts 2 .

Now, if we apply the influence model:

$$
y_{x_{1}}^{(\infty)}=V y_{x_{1}}^{(1)}
$$

and we obtain the following solution for the alternative $x_{1}$ :

$$
y_{x_{1}}^{(\infty)}=\left(\begin{array}{c}
0.48 \\
0.37 \\
0.59
\end{array}\right)
$$

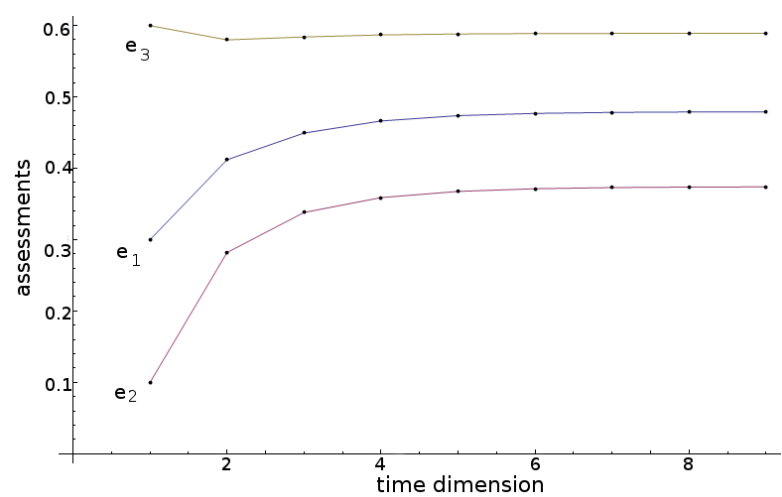

Fig. 3 Evolution of the alternative $x_{1}$

Looking the Figure 3 we can study the evolution of alternative $x_{1}$. So, the expert $e_{1}$ starts with an assessment of 0.3 about the alternative $x_{1}$, experts $e_{2}$ starts with 0.1 , and $e_{3}$ with 0.6 . The final experts' opinions are $0.48,0.37$, and 0.59 respectively. As can be seen, the most influential expert is $e_{3}$ whose opinion scarcely changes, and on the other hand, experts $e_{1}$ and $e_{2}$ are greatly affect by the opinion of $e_{3}$ that is the reason why their opinion change largely from 0.3 and 0.1 to 0.48 and 0.37 respectively.

\subsection{Obtaining the solution}

The aim of this final phase is to obtain the solution of the GDM problem, i.e., a global utility vector that provides us with an ordering vector of alternatives from the best one to the worst one.

To obtain the solution, the utility vectors obtained in the previous phase are aggregated by means of an IIOWA operator. This operator requires a vector of importance degrees. In this model, it seems logical to use 
the experts' consistency degree as the order inducing values. We assume that experts who show more consistency in their assessment are supposed to be more reliable, and therefore they should have a greater weight in the aggregation.

In order to compute the experts' consistency degree, vector $W_{c}$, we use the $U$-consistency level for each experts' preference relation by using the formula reviewed in section 2.2:

$$
W_{c}=\left(U C L_{P_{1}}, \cdots, U C L_{P_{m}}\right)
$$

Where $U C L_{P_{i}}$ is the $U$ - consistency level of the preference relation $P_{i}$ that has been provided by the expert $e_{i}$.

Once we have calculated this vector, we obtain the final solution, $U_{s}$, as follows:

$$
U_{s}=\Phi_{W}^{I}\left(U_{1}^{(\infty)}, \cdots, U_{m}^{(\infty)}\right)=\left(\begin{array}{c}
u_{s}^{1} \\
\cdots \\
u_{s}^{n}
\end{array}\right)
$$

where the best alternative has the greater value, and worst one, the smaller one.

\section{Example of Application of the Influence Model in Group Decision Making}

Let $E=\left\{e_{1}, e_{2}, e_{3}, e_{4}, e_{5}, e_{6}, e_{7} . e_{8}\right\}$ be a set of eight experts and six alternatives $X=\left\{x_{1}, x_{2}, x_{3}, x_{4}, x_{5}, x_{6}\right\}$. To show the model, we carry the following phases.

\section{Phase 1: Providing the information}

There are eight experts: $E=\left\{e_{1}, e_{2}, e_{3}, e_{4}, e_{5}, e_{6}, e_{7}, e_{8}\right\}$ with their initial preference relations respective (see Table 1),

Now we check the consistency and improve the quality of the information provided (see Table 2)

\section{Phase 2: Modeling influence}

Preference relations are transformed into utility vectors. To do so, the dominance degree is computed. We use the weighting vector $W_{O W A}=(0.41,0.17,0.13,0.11$, $0.096,0.087)$ calculated with the linguistic quantifier "most of" defined by $Q(r)=r^{1 / 2}$.

Finally, the utility vectors obtained from the preference relations are the followings:

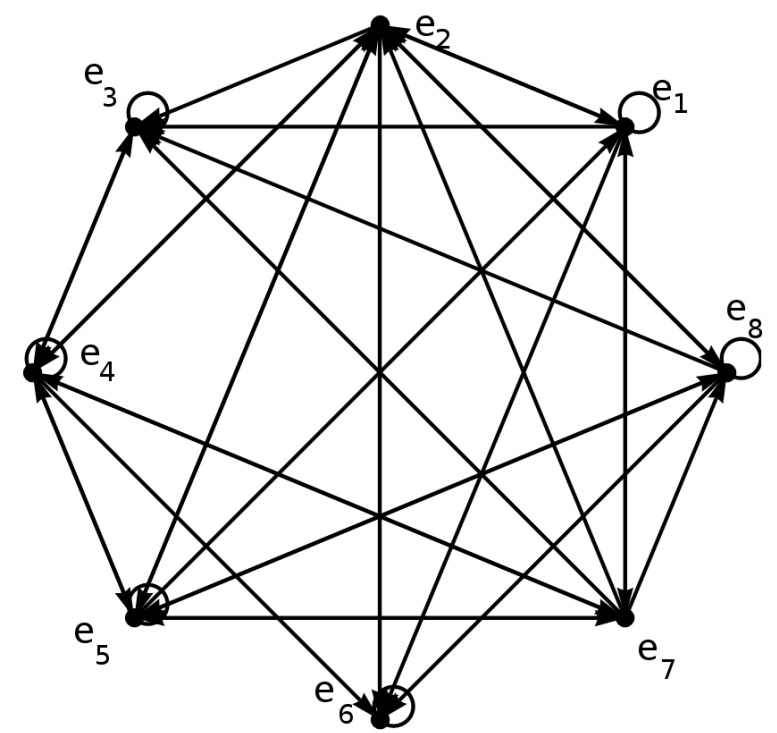

Fig. 4 Matrix W of interpersonal influences

$$
\begin{aligned}
& U_{1}=\left(\begin{array}{l}
0.74 \\
0.84 \\
0.66 \\
0.31 \\
0.58 \\
0.49
\end{array}\right) U_{2}=\left(\begin{array}{l}
0.84 \\
0.66 \\
0.58 \\
0.74 \\
0.31 \\
0.49
\end{array}\right) U_{3}=\left(\begin{array}{c}
0.61 \\
0.48 \\
0.86 \\
0.81 \\
0.7 \\
0.27
\end{array}\right) U_{4}=\left(\begin{array}{l}
0.46 \\
0.84 \\
0.58 \\
0.31 \\
0.77 \\
0.66
\end{array}\right) \\
& U_{5}=\left(\begin{array}{l}
0.70 \\
0.68 \\
0.88 \\
0.56 \\
0.55 \\
0.29
\end{array}\right) U_{6}=\left(\begin{array}{l}
0.45 \\
0.73 \\
0.69 \\
0.57 \\
0.45 \\
0.54
\end{array}\right) U_{7}=\left(\begin{array}{l}
0.76 \\
0.65 \\
0.58 \\
0.57 \\
0.49 \\
0.49
\end{array}\right) U_{8}=\left(\begin{array}{l}
0.56 \\
0.42 \\
0.60 \\
0.47
\end{array}\right) .
\end{aligned}
$$

Each expert is supposed to talk with each other. Among them, there exists an influence network that represents the degree in which one expert's opinions is influenced by the other ones. This influence network is represented by the matrix $W$ and graphically described in Figure 4),

$$
W=\left(\begin{array}{cccccccc}
0.2 & 0.1 & 0.25 & 0 & 0 & 0.15 & 0.3 & 0 \\
0.1 & 0 & 0.18 & 0.05 & 0.34 & 0.24 & 0 & 0.09 \\
0 & 0 & 0.5 & 0.5 & 0 & 0 & 0 & 0 \\
0 & 0.1 & 0.23 & 0.34 & 0.14 & 0.09 & 0.1 & 0 \\
0.2 & 0.08 & 0 & 0.021 & 0.05 & 0 & 0.049 & 0.6 \\
0 & 0 & 0 & 0 & 0 & 1 & 0 & 0 \\
0.2 & 0.09 & 0.05 & 0.32 & 0.04 & 0 & 0 & 0.3 \\
0 & 0.07 & 0.27 & 0 & 0.5 & 0.04 & 0 & 0.12
\end{array}\right) .
$$

After applying the influence model, the utility vectors obtained are the following: 


$$
\begin{aligned}
& P_{1}=\left(\begin{array}{llllll}
0.50 & 0.40 & 0.60 & 0.90 & 0.70 & 0.80 \\
0.60 & 0.50 & 0.70 & 1.00 & 0.80 & 0.90 \\
0.40 & 0.30 & 0.50 & 0.80 & 0.60 & 0.70 \\
0.10 & 0.00 & 0.20 & 0.50 & 0.30 & 0.40 \\
0.30 & 0.20 & 0.40 & 0.70 & 0.50 & 0.60 \\
0.20 & 0.10 & 0.30 & 0.60 & 0.40 & 0.50
\end{array}\right) \\
& P_{4}=\left(\begin{array}{llllll}
0.50 & 0.10 & 0.36 & 0.69 & 0.16 & 0.26 \\
0.90 & 0.50 & 0.84 & 0.95 & 0.62 & 0.76 \\
0.64 & 0.16 & 0.50 & 0.80 & 0.25 & 0.39 \\
0.31 & 0.05 & 0.20 & 0.50 & 0.08 & 0.14 \\
0.84 & 0.38 & 0.75 & 0.92 & 0.50 & 0.66 \\
0.74 & 0.24 & 0.61 & 0.86 & 0.34 & 0.50
\end{array}\right) \\
& P_{7}=\left(\begin{array}{llllll}
0.50 & 0.55 & 0.45 & 0.25 & 0.70 & 0.30 \\
0.45 & 0.50 & 0.70 & 0.85 & 0.40 & 0.80 \\
0.55 & 0.30 & 0.50 & 0.65 & 0.70 & 0.60 \\
0.75 & 0.15 & 0.35 & 0.50 & 0.95 & 0.60 \\
0.30 & 0.60 & 0.30 & 0.05 & 0.50 & 0.85 \\
0.70 & 0.20 & 0.40 & 0.40 & 0.15 & 0.50
\end{array}\right)
\end{aligned}
$$

$$
P_{2}=\left(\begin{array}{llllll}
0.50 & 0.70 & 0.80 & 0.60 & 1.00 & 0.90 \\
0.30 & 0.50 & 0.60 & 0.40 & 0.80 & 0.70 \\
0.20 & 0.40 & 0.50 & 0.30 & 0.70 & 0.60 \\
0.40 & 0.60 & 0.70 & 0.50 & 0.90 & 0.80 \\
0.00 & 0.20 & 0.30 & 0.10 & 0.50 & 0.40 \\
0.10 & 0.30 & 0.40 & 0.20 & 0.60 & 0.50
\end{array}\right)
$$

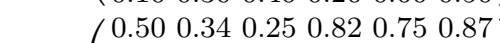

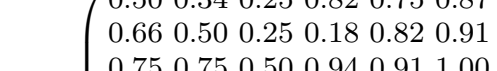$$
P_{5}=
$$
$\begin{array}{lllllll}0.75 & 0.75 & 0.50 & 0.94 & 0.91 & 1.00\end{array}$ $\begin{array}{lllllll}0.18 & 0.82 & 0.06 & 0.50 & 0.34 & 0.75\end{array}$ $\begin{array}{lllllll}0.25 & 0.18 & 0.09 & 0.66 & 0.50 & 0.82\end{array}$ $\left(\begin{array}{llllll}0.13 & 0.09 & 0.00 & 0.25 & 0.18 & 0.50\end{array}\right)$ $P_{8}=\left(\begin{array}{llllll}0.50 & 0.70 & 0.75 & 0.95 & 0.60 & 0.85 \\ 0.30 & 0.50 & 0.55 & 0.80 & 0.40 & 0.65 \\ 0.25 & 0.45 & 0.50 & 0.70 & 0.60 & 0.45 \\ 0.05 & 0.20 & 0.30 & 0.50 & 0.85 & 0.40 \\ 0.40 & 0.60 & 0.40 & 0.15 & 0.50 & 0.75 \\ 0.15 & 0.35 & 0.55 & 0.60 & 0.25 & 0.50\end{array}\right)$.
$P_{3}=\left(\begin{array}{llllll}0.50 & 0.69 & 0.12 & 0.20 & 0.36 & 0.90 \\ 0.31 & 0.50 & 0.06 & 0.10 & 0.20 & 0.80 \\ 0.88 & 0.94 & 0.50 & 0.64 & 0.80 & 0.98 \\ 0.80 & 0.90 & 0.36 & 0.50 & 0.69 & 0.97 \\ 0.64 & 0.80 & 0.20 & 0.31 & 0.50 & 0.94 \\ 0.10 & 0.20 & 0.02 & 0.03 & 0.06 & 0.50\end{array}\right)$

$P_{6}=\left(\begin{array}{llllll}0.50 & 0.13 & 0.18 & 0.34 & 0.75 & 0.09 \\ 0.87 & 0.50 & 0.66 & 0.82 & 0.91 & 0.25 \\ 0.82 & 0.34 & 0.50 & 0.75 & 0.87 & 0.82 \\ 0.66 & 0.18 & 0.25 & 0.50 & 0.75 & 0.91 \\ 0.25 & 0.09 & 0.13 & 0.25 & 0.50 & 0.97 \\ 0.91 & 0.75 & 0.18 & 0.09 & 0.03 & 0.50\end{array}\right)$
Table 1 Experts' initial opinions

$$
\begin{aligned}
P_{1}^{\prime} & =\left(\begin{array}{llllll}
0.50 & 0.27 & 0.64 & 0.89 & 0.74 & 0.82 \\
0.73 & 0.50 & 0.80 & 0.92 & 0.85 & 0.89 \\
0.36 & 0.20 & 0.50 & 0.85 & 0.62 & 0.74 \\
0.11 & 0.08 & 0.15 & 0.50 & 0.20 & 0.27 \\
0.26 & 0.15 & 0.38 & 0.80 & 0.50 & 0.64 \\
0.18 & 0.11 & 0.26 & 0.73 & 0.36 & 0.50
\end{array}\right) \\
P_{4}^{\prime} & =\left(\begin{array}{llllll}
0.50 & 0.10 & 0.36 & 0.69 & 0.16 & 0.27 \\
0.90 & 0.50 & 0.83 & 0.95 & 0.63 & 0.76 \\
0.64 & 0.17 & 0.50 & 0.79 & 0.25 & 0.39 \\
0.31 & 0.05 & 0.21 & 0.50 & 0.08 & 0.14 \\
0.84 & 0.37 & 0.75 & 0.92 & 0.50 & 0.65 \\
0.73 & 0.24 & 0.61 & 0.86 & 0.35 & 0.50
\end{array}\right) \\
P_{7}^{\prime}= & \left(\begin{array}{llllll}
0.50 & 0.30 & 0.40 & 0.45 & 0.51 & 0.66 \\
0.70 & 0.50 & 0.53 & 0.45 & 0.73 & 0.68 \\
0.60 & 0.47 & 0.50 & 0.40 & 0.54 & 0.66 \\
0.55 & 0.55 & 0.60 & 0.50 & 0.44 & 0.60 \\
0.49 & 0.27 & 0.46 & 0.56 & 0.50 & 0.37 \\
0.34 & 0.32 & 0.34 & 0.40 & 0.63 & 0.50
\end{array}\right)
\end{aligned}
$$

$P_{8}^{\prime}=\left(\begin{array}{cccccc}0.50 & 0.75 & 0.75 & 0.72 & 0.77 & 0.82 \\ 0.25 & 0.50 & 0.55 & 0.62 & 0.59 & 0.65 \\ 0.25 & 0.45 & 0.50 & 0.60 & 0.46 & 0.67 \\ 0.28 & 0.38 & 0.40 & 0.50 & 0.20 & 0.44 \\ 0.23 & 0.41 & 0.54 & 0.80 & 0.50 & 0.50 \\ 0.18 & 0.35 & 0.33 & 0.56 & 0.50 & 0.50\end{array}\right)$.
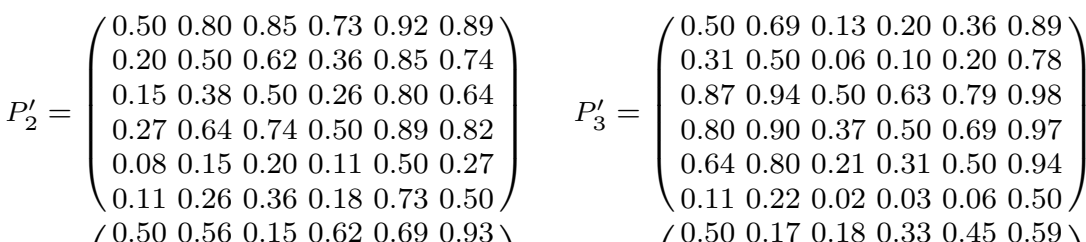

$$
\begin{aligned}
& P_{2}^{\prime}=\left(\begin{array}{llllll}
0.50 & 0.80 & 0.85 & 0.73 & 0.92 & 0.89 \\
0.20 & 0.50 & 0.62 & 0.36 & 0.85 & 0.74 \\
0.15 & 0.38 & 0.50 & 0.26 & 0.80 & 0.64 \\
0.27 & 0.64 & 0.74 & 0.50 & 0.89 & 0.82 \\
0.08 & 0.15 & 0.20 & 0.11 & 0.50 & 0.27 \\
0.11 & 0.26 & 0.36 & 0.18 & 0.73 & 0.50
\end{array}\right) \quad P_{3}^{\prime}=\left(\begin{array}{llllll}
0.50 & 0.69 & 0.13 & 0.20 & 0.36 & 0.89 \\
0.31 & 0.50 & 0.06 & 0.10 & 0.20 & 0.78 \\
0.87 & 0.94 & 0.50 & 0.63 & 0.79 & 0.98 \\
0.80 & 0.90 & 0.37 & 0.50 & 0.69 & 0.97 \\
0.64 & 0.80 & 0.21 & 0.31 & 0.50 & 0.94 \\
0.11 & 0.22 & 0.02 & 0.03 & 0.06 & 0.50
\end{array}\right) \\
& P_{6}^{\prime}=\left(\begin{array}{llllll}
0.50 & 0.17 & 0.18 & 0.33 & 0.45 & 0.59 \\
0.83 & 0.50 & 0.47 & 0.61 & 0.71 & 0.82 \\
0.82 & 0.53 & 0.50 & 0.60 & 0.70 & 0.61 \\
0.67 & 0.39 & 0.40 & 0.50 & 0.62 & 0.46 \\
0.55 & 0.29 & 0.30 & 0.38 & 0.50 & 0.31 \\
0.41 & 0.18 & 0.39 & 0.54 & 0.69 & 0.50
\end{array}\right)
\end{aligned}
$$

Table 2 Experts' opinions more consistent

$$
\begin{array}{r}
U_{1}^{\prime}=\left(\begin{array}{l}
0.60 \\
0.69 \\
0.72 \\
0.53 \\
0.61 \\
0.45
\end{array}\right) U_{2}^{\prime}=\left(\begin{array}{l}
0.56 \\
0.65 \\
0.73 \\
0.58 \\
0.60 \\
0.45
\end{array}\right) U_{3}^{\prime}=\left(\begin{array}{l}
0.58 \\
0.56 \\
0.80 \\
0.70 \\
0.70 \\
0.35
\end{array}\right) U_{4}^{\prime}=\left(\begin{array}{l}
0.52 \\
0.72 \\
0.67 \\
0.48 \\
0.69 \\
0.53
\end{array}\right) \\
U_{5}^{\prime}=\left(\begin{array}{l}
0.60 \\
0.64 \\
0.73 \\
0.57 \\
0.62 \\
0.42
\end{array}\right) U_{6}^{\prime}=\left(\begin{array}{l}
0.57 \\
0.73 \\
0.69 \\
0.57 \\
0.45 \\
0.54
\end{array}\right) U_{7}^{\prime}=\left(\begin{array}{l}
0.61 \\
0.62 \\
0.55 \\
0.64 \\
0.46
\end{array}\right) U_{8}^{\prime}=\left(\begin{array}{l}
0.72 \\
0.58 \\
0.63 \\
0.42
\end{array}\right)
\end{array}
$$

\section{Phase 3: Exploitation}

In the final phase we aggregate the utility vectors obtained in the previous step by means of an I-IOWA operator. In order to accomplish this phase, we need to compute vector $W_{c}$. This vector is computed from the experts' consistency degree and it is calculated as we explained in section 2.2 :

$$
W_{c}=(0.94,0.94,0.99,1,0.86,0.75,0.76,0.85) .
$$

This vector is used as a weighting vector to compute the solution.

The global opinion obtained after the aggregation is:

$$
U_{s}=\left(\begin{array}{c}
0.53 \\
0.68 \\
0.71 \\
0.57 \\
0.57 \\
0.48
\end{array}\right)
$$

From this vector, the order of the alternatives, from the best one to worst one is:

$$
\left\{x_{3}, x_{2}, x_{4}, x_{5}, x_{1}, x_{6}\right\}
$$




\section{Conclusion}

In this proposal we have presented a novel approach to model the experts' interaction in a group decision making problem.

GDM classical models do not take into account the influence concept among experts to carry out the decision making. However, in real group decision making problems, the influence may play a key role to accomplish this task. For example, popular people in Social Networks are known to be able to stablish trends or styles.

The new model takes into account the relationship among experts to create a social network. This social network allows us to represent the experts' social influence network. Moreover, because of the use of this methodology, we can infer and study the evolution of the experts' opinions as well as predicting the solution of the group decision making problem. In the future, we will study a better representation for the experts' social network to make easier its use. Besides, considering that Social Networks have a very relevant role in many areas of the modern society, this proposal could be very useful to address real problems in which it is required to predict the final result of a group decision making problem from the people's initial opinions without carrying out the GDM process. On the other hand, we also want to study how modelling the influence in linguistic contexts [24,44] and in heterogeneous decision making frameworks $[29,31]$.

Acknowledgements This research work has been supported with Feder funding by the research project of Education Ministery TIN2013-40658-P.

\section{Compliance with Ethical Standards:}

Funding: This study was funded by the research project of Education Ministery TIN2013-40658-P.

Conflict of Interest: Author Luis G. Pérez declares that he has no conflict of interest. Author Francisco Mata declares that he has no conflict of interest. Author Francisco Chiclana declares that he has no conflict of interest. Author Gang Kou declares that he has no conflict of interest. Author Enrique Herrera-Viedma declares that he has no conflict of interest.

Ethical approval: This article does not contain any studies with human participants or animals performed by any of the authors.

\section{Appendix}

\section{A Fuzzy Quantifiers and Their Use To Model Fuzzy Majority}

Fuzzy majority is a soft majority concept expressed by a fuzzy quantifier. This fuzzy quantifier is manipulated by means of a fuzzy logic-based calculus of linguistically quantified propositions. Hence, the use of fuzzy-majority-guided aggregation operators allows us to incorporate the concept of majority into the computation of the solution.

Quantifier are used to represent the amount of items satisfying a given predicate. Classic logic is restricted to two quantifiers, there exists and for all, however, human discourse is much richer and more diverse. In order to provide a more flexible knowledge representation tool, Zadeh introduced the concept of fuzzy quantifiers [51].

Zadeh suggested that the semantics of a fuzzy quantifier can be captured by using fuzzy subsets for its representation. Moreover, he differentiated between two types of fuzzy quantifiers: absolute and relative ones. In this model, we have focused on relative quantifiers, such as most, at least half, etc., since they can simbolize any quantifier of natural language. These quantifiers can be represented by fuzzy subsets of the unit interval $[0,1]$. For any $r \in[0,1], Q(r)$ indicates the degree in which the proportion $\mathrm{r}$ is compatible with the meaning of the quantifier it represents.

A relative quantifier $Q:[0,1] \rightarrow[0,1]$ satisfies

$$
Q(0)=0 \exists r \in[0,1] \text { such that } Q(r)=1
$$

In [47], Yager identified two categories of relative quantifiers: RIM quantifiers such as all, most, many, at least $\alpha$; and regular decreasing monotone (RDM) quantifiers such as at most one, few, at most $\alpha$, being the former one, the category used in this proposal.

A RIM quantifier satisfies

$$
\forall a, b \text { if } a>b \text { then } Q(a) \geq Q(b) .
$$

Yager in [47] considers the parameterized family of RIM quantifiers

$$
Q(r)=r^{\alpha}, \alpha \geq 0
$$

When this family of RIM quantifiers is used with OWA and IOWA operators, $\alpha<1$ in order to associate high weighting values with high consistent ones. In particular, in this paper, we use the RIM function $Q(r)=r^{1 / 2}$.

\section{B Example of Group Decision Making}

Let $E=\left\{e_{1}, e_{2}, e_{3}, e_{4}\right\}$ be the group of four experts and $X=$ $\left\{x_{1}, x_{2}, x_{3}, x_{4}\right\}$ be the set of four alternatives. This group of experts, $E$, express their preferences about the set of alternatives, $X$, by means of fuzzy preference relations, $\left\{P_{1}, P_{2}, P_{3}, P_{4}\right\}$, $P_{k}=\left[p_{i j}^{k}\right], p_{i j}^{k} \in[0,1]$, which are additive reciprocal. $X$ :

Consider the following preferences over the set of alternatives

$$
\begin{aligned}
& P_{1}=\left(\begin{array}{llll}
0.5 & 0.3 & 0.7 & 0.1 \\
0.7 & 0.5 & 0.6 & 0.6 \\
0.3 & 0.4 & 0.5 & 0.2 \\
0.9 & 0.4 & 0.8 & 0.5
\end{array}\right) P_{2}=\left(\begin{array}{llll}
0.5 & 0.4 & 0.6 & 0.2 \\
0.6 & 0.5 & 0.7 & 0.4 \\
0.4 & 0.3 & 0.5 & 0.1 \\
0.8 & 0.6 & 0.9 & 0.5
\end{array}\right) \\
& P_{3}=\left(\begin{array}{cccc}
0.5 & 0.5 & 0.7 & 0 \\
0.5 & 0.5 & 0.8 & 0.4 \\
0.3 & 0.2 & 0.5 & 0.2 \\
1 & 0.6 & 0.8 & 0.5
\end{array}\right) P_{4}=\left(\begin{array}{llll}
0.5 & 0.4 & 0.7 & 0.8 \\
0.6 & 0.5 & 0.4 & 0.3 \\
0.3 & 0.6 & 0.5 & 0.1 \\
0.2 & 0.7 & 0.9 & 0.5
\end{array}\right),
\end{aligned}
$$


and their respective expert's importance $I=\{0.75,1,0.5,0.25\}$.

We use the fuzzy linguistic quantifier 'most of' defined by $Q(r)=r^{1 / 2}$ [47], with its corresponding weighting vector $W=$ $(0.5,0.21,0.16,0.13)$. By using the I-IOWA operator, the following collective preference relation is computed:

$$
P_{c}=\left(\begin{array}{cccc}
0.5 & 0.4 & 0.65 & 0.23 \\
0.6 & 0.5 & 0.65 & 0.43 \\
0.35 & 0.35 & 0.5 & 0.14 \\
0.77 & 0.57 & 0.86 & 0.5
\end{array}\right) .
$$

Now, if we use the quantifier-guided dominance degree, $Q G D D_{i}$ with the weighting vector $W_{Q G D D}=(0.5,0.21,0.16,0.13)$, the following utility vector is obtained:

$$
U^{c}=\left(\begin{array}{c}
0.52 \\
0.59 \\
0.4 \\
0.75
\end{array}\right),
$$

and therefore the final solution is:

$$
\left(x_{4}, x_{2}, x_{1}, x_{3}\right) \text {, }
$$

i.e. $x_{4}$ is the preferred alternative, $x_{2}$ is the second one, etc.

\section{References}

1. Alonso, S., Cabrerizo, F.J., Chiclana, F., Herrera, F., Herrera-Viedma, E.: Group decision making with incomplete fuzzy linguistic preference relations. International Journal of Intelligent Systems 24(2), 201-222 (2009)

2. Alonso, S., Herrera-Viedma, E., Chiclana, F., Herrera, F.: A web based consensus support system for group decision making problems and incomplete preferences. Information Sciences 180(23), 4477-4495 (2010)

3. Bezdek, J.C., Spillman, B., Spillman, R.: A fuzzy relation space for group decision theory. Fuzzy Sets and Systems $\mathbf{1}(4), 255-268$ (1978)

4. Cabrerizo, F., Chiclana, F., Al-Hmouz, R., Morfeq, A., Balamash, A., Herrera-Viedma, E.: Fuzzy decision making and consensus: challenges. Journal of Intelligent \& Fuzzy Systems (In press)

5. Cabrerizo, F., Moreno, J., Pérez, I., Herrera-Viedma, E.: Analyzing consensus approaches in fuzzy group decision making: advantages and drawbacks. Soft Computing 14(5), 451-463 (2010)

6. Calza, F., Gaeta, M., Loia, V., Orciuoli, F., Piciocchi, P., Rarità, L., Spohrer, J., Tommasetti, A.: Fuzzy consensus model for governance in smart service systems. Procedia Manufacturing 3, 3567-3574 (2015)

7. Chiclana, F., Herrera-Viedma, E., Alonso, S., Herrera, F.: Consistency of reciprocal preference relations. In: 2007 IEEE International Conference on Fuzzy Systems, FUZZY (2007)

8. Chiclana, F., Herrera-Viedma, E., Alonso, S., Herrera, F.: A note on the estimation of missing pairwise preference values: A uninorm consistency based method. International Journal of Uncertainty, Fuzziness and Knowlege-Based Systems 16(SUPPL.2), 19-32 (2008)

9. Chiclana, F., Herrera-Viedma, E., Alonso, S., Herrera, F.: Cardinal consistency of reciprocal preference relations: A characterization of multiplicative transitivity. IEEE Transactions on Fuzzy Systems 17(1), 14-23 (2009)

10. Chiclana, F., Herrera-Viedma, E., Herrera, F., Alonso, S.: Some induced ordered weighted averaging operators and their use for solving group decision-making problems based on fuzzy preference relations. European Journal of Operational Research 182(1), 383-399 (2007)
11. Ciasullo, M.V., Gaeta, M., Monetta, G., Rarità, L.: Ecultural value co-creation. A proposed model for the heritage management. In: Proceedings of 18th Toulon-Verona International Conference, "Excellence in Services", vol. U, pp. 139-158 (2015)

12. Dubois, D.J., Prade, H.: Fuzzy Sets and Systems: Theory and Application. Academic Press, New York (1980)

13. French, J.R.: A formal theory of social power. Psychological review 63(3), 181-194 (1956)

14. Friedkin, N.E., Johnsen, E.C.: Social influence and opinions (1990)

15. Friedkin, N.E., Johnsen, E.C.: Social influence networks and opinion change (1999)

16. Gong, Z., Xu, X., Zhang, H., Ozturk, U.A., Herrera-Viedma, E., Xu, C.: The consensus models with interval preference opinions and their economic interpretation. Omega 55, 81$90(2015)$

17. Harary, F.: A criterion for unanimity in French's theory of social power, pp. 168-182. Studies in social power. Ann Arbor: University of Michigan, Oxford, England (1959)

18. Kacprzyk, J.: Group decision making with a fuzzy linguistic majority. Fuzzy Sets and Systems 18(2), 105-118 (1986)

19. Kacprzyk, J., Fedrizzi, M., Nurmi, H.: Consensus Under Fuzziness. Kluwer Academic Publishers (1997)

20. Kacprzyk, J., Roubens, M.: Non-conventional preference relations in decision making. Springer, Berlin (1988)

21. Kitainik, L.: Fuzzy Decision Procedures with Binary Relations: Towards a Unified Theory. Springer (1993)

22. Klement, E.P., Mesiar, R., Pap, E.: On the relationship of associative compensatory operators to triangular norms and conorms. International Journal of Uncertainty, Fuzziness and Knowlege-Based Systems 4(2), 129-144 (1996)

23. Luce, R.D., Suppes, P.: Preferences, utility and subject probability, pp. 249-410. Handbook of Mathematical Psychology, ,Volumen III. Wiley, New York (1965)

24. Massanet, S., Riera, J.V., Torrens, J., Herrera-Viedma, E.: A new linguistic computational model based on discrete fuzzy numbers for computing with words. Information Sciences 258, 277-290 (2014)

25. Mata, F., Martínez, L., Herrera-Viedma, E.: An adaptive consensus support model for group decision making problems in a multi-granular fuzzy linguistic context. IEEE Transactions on Fuzzy Systems 17(2), 279-290 (2009)

26. Mata, F., Pérez, L., Zhou, S.M., Chiclana, F.: Type-1 owa methodology to consensus reaching processes in multigranular linguistic contexts. Knowledge-Based Systems $\mathbf{5 8}(0), 11-22(2014)$

27. Mitchell, H.B., Estrakh, D.D.: A modified owa operator and its use in lossless dpcm image compression. International Journal of Uncertainty, Fuzziness and Knowledge-Based Systems 5, 429-436 (1997)

28. Morente-Molinera, J., Al-hmouz, R., Morfeq, A., Balamash, A., Herrera-Viedma, E.: A decision support system for decision making in changeable and multi-granular fuzzy linguistic contexts. J. of Mult.-Valued Logic \& Soft Computing (In press)

29. Morente-Molinera, J., Pérez, I., Ureña, M., Herrera-Viedma, E.: Building and managing fuzzy ontologies with heterogeneous linguistic information. Knowledge-Based Systems (2015, In press)

30. Nurmi, H.: Approaches to collective decision making with fuzzy preference relations. Fuzzy Sets and Systems 6(3), 249$259(1981)$

31. Pérez, I.J., Cabrerizo, F.J., Alonso, S., Herrera-Viedma, E. A new consensus model for group decision making problems with non-homogeneous experts. Systems, Man, and Cybernetics: Systems, IEEE Transactions on 44(4), 494-498 (2014) 
32. Pérez, I.J., Cabrerizo, F.J., Herrera-Viedma, E.: A mobile decision support system for dynamic group decision-making problems. IEEE Transactions on Systems, Man, and Cybernetics Part A: Systems and Humans 40(6), 1244-1256 (2010)

33. Pérez, L., Mata, F., Chiclana, F.: Social network decision making with linguistic trustworthiness based induced owa operators. International Journal of Intelligent Systems 29(12), 1117-1137 (2014)

34. Pérez-Asurmendi, P., Chiclana, F.: Linguistic majorities with difference in support. Applied Soft Computing 18, 196-208 (2014)

35. Roubens, M.: Fuzzy sets and decision analysis. Fuzzy Sets and Systems 90(2), 199-206 (1997)

36. Saaty, T.: The Analytic Hierarchy Process. McGraw-Hill, New york (1980)

37. Scott, J.P., Carrington, P.J.: The SAGE Handbook of Social Network Analysis. SAGE, London (2011)

38. Seo, F., Sakawa, M.: Fuzzy multiattribute utility analysis for collective choice. IEEE Transactions on Systems, Man and Cybernetics 15(1), 45-53 (1985)

39. Tanino, T.: Fuzzy preference orderings in group decision making. Fuzzy Sets and Systems 12(2), 117-131 (1984)

40. Tanino, T.: Fuzzy preference relations in group decision making, pp. 54-71. Non-Conventional Preference Relations in Decision Making. Springer-Verlag, Berlin (1988)

41. Tanino, T.: On group decision making under fuzzy preferences, pp. 172-185. Multiperson Decision Making Using Fuzzy Sets and Possibility Theory. Kluwer Academic Publisher, Dordrecht (1990)

42. Ureña, R., Chiclana, F., Morente-Molinera, J., HerreraViedma, E.: Managing incomplete preference relations in decision making: A review and future trends. Information Sciences 302, $14-32$ (2015)

43. Wasserman, S., Faust, K.: Social Network Analysis: Methods and Applications. Cambridge University Press (1994)

44. Wu, J., Chiclana, F., Herrera-Viedma, E.: Trust based consensus model for social network in an incomplete linguistic information context. Applied Soft Computing 35, 827 - 839 (2015)

45. Yager, R.R.: Quantifiers in the formulation of multiple objective decision functions. Information Sciences 31(2), 107-139 (1983)

46. Yager, R.R.: On ordered weighted averaging aggregation operators in multicriteria decision making. IEEE Transactions on Systems, Man and Cybernetics 18(1), 183-190 (1988)

47. Yager, R.R.: Quantifier guided aggregation using OWA operators. International Journal of Intelligent Systems 11(1), 49-73 (1996)

48. Yager, R.R.: Induced aggregation operators. Fuzzy Sets and Systems 137, 59-69 (2003)

49. Yager, R.R., Filev, D.: Operations for granular computing: Mixing words and numbers. In: IEEE International Conference on Fuzzy Systems, vol. 1, pp. 123-128 (1998)

50. Yager, R.R., Filev, D.: Induced ordered weighted averaging operators. IEEE Transaction on Systems, Man and Cybernetics 29, 141-150 (1999)

51. Zadeh, L.A.: A computational approach to fuzzy quantifiers in natural languages. Computers and Mathematics with Applications 9(1), 149-184 (1983) 